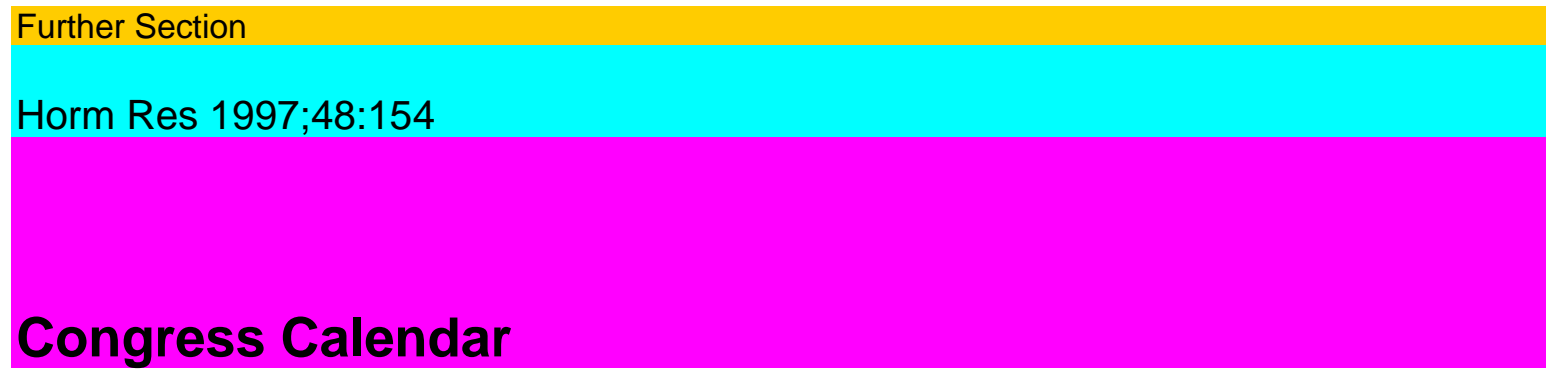

\title{
30.08.-03.09.1997 Munich
}

Germany

04.09.-05.09.1997 Munich

Germany

04.09.-06.09.1997 Berlin

Germany

04.09.-06.09.1997 Copenhagen

Denmark

04.09-07.09.1997 San Francisco, Calif.

USA

06.09.1997 Kiel

Germany

11.09.-14.09.1997 Karlsburg/Greif/swald

Germany

17.09.-22.09.1997 Jerusalem

Israel

28.09.-02.10.1997 Sydney

Australia

29.09.-01.10.1997 Canberra

Australia

10.10.-14.10.1997 Aspen, Colo.

USA

15.10.-18.10.1997 Stuttgart

Germany

21.10.-24.10.1997 Tokyo

Japan

24th Annual Meeting of the European Thyroid Association

1st International Symposium on Genetics of Thyroid Disease

Diabetic Angiopathy in Children

4th International hGH Symposium on GH Deficiency in Adults

Meeting of the Growth Hormone Research Society

7th Thyroid Symposium (Schilddrüse-Symposium)

16th Karlsburg Symposium on Problems of Diabetes - New Aspects of Diabetes Therapy

35th Annual Meeting of the European Association for the Study of Diabetes

Annual Scientific Meeting of the Endocrine Society of Australia

Joint Meeting of the Endocrine Society of Australia and New Zealand Society of

Endocrinology

3rd International Workshop on Resistance to Thyroid Hormone

Intensivkurs für klinische Endokrinologie 
4th International Symposium on Insulin-like Growth Factors

Contact: Anton Kössl, INTERPLAN Kongressdienst, Sophienstrasse 1, D-80333 Munich,

Germany Tel: +49 89 594492; Fax: +49 89591610

Contact: Prof. Dr. Josef Köhrle, Röntgenring 11, Medizinische Polikünik der Univ.,

Klinische Forschergruppe, D-97070 Würzburg, Germany Fax:+49 931565-37

Contact: Prof. Dr. Bruno Weber, Kinderklinik, Virchow-Klinikum, Augustenburger Platz 1,

D-13353 Berlin, Germany Tel: +49 303035 4219; Fax: +49 3030354638

Contact: Prof. Jens S. Christiansen, 2nd Univ. Clinic, Internal Medicine, Kommunehospitalet,

DK-8000 Aarhus C, Denmark Tel: +45 894 92013; Fax: +45 89492010

Contact: Prof. Raymond Híntz,

Dept. of Pediatrics,

Stanford University School of Medicine,

Stanford, CA 94305-5119, USA

Tel: +1 415723 5791; Fax: +1 4157235791

Contact: Prof. Dr. med. E. Henze, Arnold-Heller-Strasse 9, Klinik für Nuklearmedizin,

Radiol. Univ-Klinik, D-24105 Kiel, Germany Tel: +49 431597 3076; Fax: +49 4315973065

Contact: Dr. S. Schmidt, Institut für Diabetes,

Greifswalder Strasse 11 a,

D-17495 Karlsburg, Germany

Tel: +49 38355 701300; Fax: +49 38355701558

Contact: Helmut Schrank, DER Deutsches Reisebüro,

Emil-von-Behring-Strasse 6,

D-60439 Frankfurt am Main, Germany

Tel: +4969 9588 3610/3626; Fax: +4969 95883608

Contact: Dr. D.J. Toplíss, Ewen Downie Metabolic Unit, Alfred Hospital, Commercial Road, Melbourne, VIC 3181, Australia Tel: +61 39276 3453; Fax: +61 392763782

Contact: Dr. K.R. Lapwood, Dept. of Physiology and Anatomy, Massey University, P.B. 11222, Palmerston North, New Zealand Tel: +64 3504483

Contact: Dr. Samuel Refetoff,

The University of Chicago (MC3090),

5841 South Maryland Avenue,

Chicago, IL 60637, USA

Tel: (773) 702 6939; Fax: (773) 7026940

Contact: Prof. Dr. Martin Grussendorf, Hospitalstrasse 34, D-70174 Stuttgart, Germany Tel:

+49711299 1999; Fax: +497112661028

Contact: Kazue Takano, MD,

Department of Medicine,

Tokyo Women's Medical College,

8-1 Kawada-cho, Shinjuku-ku, Tokyo 162, Japan

Tel: +81 33538111 ext. 38314; Fax: +81 33505929

KAKGER

E-Mail karger@,karger.ch Fax + 41613061234 http://www. karger. ch

(C) 1997 S. KargerAG, Basel

This article is also accessible online at: http://BioMedNet.com/karger 